\title{
On the Velocity of the Streamer in the Lightning Discharge
}

\author{
by \\ M. Kano and K. Murai \\ Meteorological Research Institute \\ (Received September 28, 1956)
}

\begin{abstract}
An expression of the velocity of the streamer advance along a previously ionized channel in the lightning discharge is obtained on the basis of an equation which represents the time variation of the electron density in a channel. The expression shows that, as the electron density in a previously ionized channel tends to zero, the velocity of the streamer tends, as expected, to that of the streamer advance through the virgin air (i.e. of the pilot streamer). The numerical evaluation for the velocity is obtained by the graphical method in the case of the stepped streamer, where the reasonable model of the tip field of the streamer is assumed. The result shows that the velocity of the streamer thus calculated agrees fairly well with the observed value of the velocity of the stepped streamer in the lightning discharge.
\end{abstract}

\section{Introduction}

In lightning discharges, one flash usually comprises about three strokes. One stroke consists of two stages, i.e. the leader and the return streamer, the former usually displaying the stepwise phenomenon. It is known that the mean value of the velocity with which the stepped process advances as a whole has the value between $1.0 \times 10^{7} \mathrm{~cm} / \mathrm{sec}$ and $8.0 \times 10^{7} \mathrm{~cm} / \mathrm{sec}$ in the first stroke and agrees with that of the pilot streamer which precedes the stepped streamer [1], [2]. But the individual stepped streamer follows the pilot streamer with a very high velocity which is more than $10^{9} \mathrm{~cm} / \mathrm{sec}$ [1]. In the successive strokes, the velocity of the leader processes (the dart leader streamers) are greater than that in the first stroke and are of the order of $2 \times 10^{8} \mathrm{~cm} / \mathrm{sec}$. These high velocities are due to the fact that these streamers advance along a previously ionized channel, and have so far been explained as follows:

$O A$ is the streamer stem and the shaded portions, of the previously ionized channel in front of the tip of the streamer indicates the region over which the electric field exceeds the critical value for impact ionization by electrons (Fig. 1). Let $n_{s}$ and $\bar{n}$ denote the electron density at the tip of the streamer, $A$, and the average electron density in the region $A B$ respectively, and let the distance in the direction of the field, which is assumed here to coincide with the direction of the channel, within which a single electron produces $n_{s} / \bar{n}$ electrons by collisions be 
$\delta \mathrm{cm}$. The streamer advances $d \mathrm{~cm}(d=A B)$ while a single electron advances $\delta \mathrm{cm}$. Thus the velocity of the streamer, $V$, is [3]

$$
\begin{gathered}
V=\frac{d}{\delta} \bar{v} \\
n_{s}=\bar{n} \exp \left(\int_{0}^{\delta} \alpha(z) d z\right),
\end{gathered}
$$

where $\alpha(z)$ is Townsend's first ionization coefficient, $\bar{v}$ is the mean drift velocity of the electrons in the region $A B$, and $z$ is the linear coordinate along the channel. $d$ is considered to be appreciably larger than $\delta$, so $V$ is larger than $\bar{v}$ by the factor $d / \delta$. The above mechanism is given for the negative streamer, but it essentially remains for the return streamer which is the positive streamer.

In the expression (1), however, the time variation of the electron density in the channel is not taken account of. According to (1), in order that the velocity of the streamer approaches that of the pilot streamer (i.e. $V \rightarrow \bar{v}$ ), $\delta$ must tend to d. This condition, however, seems to be too artificial. However, the streamer in question differs from the pilot streamer only by the fact that it advances along the previously ionized channel. Thus, it is desirable that the expression of the velocity of the streamer, $V$, tends to that of the pilot streamer, $\bar{v}$, if $n_{B}$ tend to zero, where $n_{B}$ is the electron density at $B . \quad n_{B}=0$ may be the condition of the virgin air in the gaseous discharge. $n_{B} \rightarrow 0$ must not be confused with $\bar{n} \rightarrow 0$.

In this paper, we tried to find a more accurate equation of the velocity of the streamer along the previously ionized region, in which the time variation of the electron density is taken account of, in place of (1). Our result shows that $V$ approaches $\bar{v}$ as $n_{B}$ approaches zero and that $V$ agrees with the observed value, if an appropriate model of the field in front of the streamer is assumed.

\section{Mechanism of the streamer advance along a previously ionized channel}

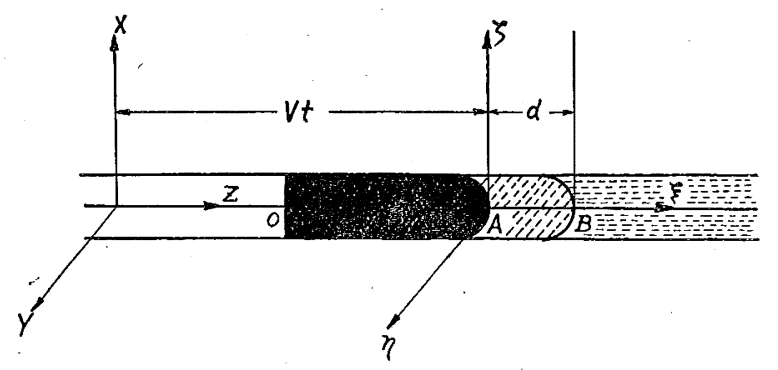

Fig. 1. The schematic diagram of the streamer stem.

Let the $(x, y, z)$ system be a coordinate system fixed in the space and the $(\zeta, \eta, \xi)$ system be a coordinate system fixed on the streamer with an origin at the tip of the streamer (Fig. 1). It is assumed that the $\xi$-axis agrees with the $z$-axis along which the streamer advances and that the tip of the streamer is at the origin of the $(x, y, z)$ coordinate when the time is zero. 
For simplicity, we shall consider from now the physical quantities on the $z$-axis alone as the representatives of this phenomenon. Then the physical quantities in the channel are the functions of $z$ and $t$ (time).

Denoting the electron density, the drift velocity of the electron and Townsend's first ionization coefficient by $n(z, t), v(z, t)$ and $\alpha(z, t)$ respectively, the equation of the time variation of the electron density in the channel becomes

$$
\frac{\partial n}{\partial t}=-v \frac{\partial n}{\partial z}+\left(\alpha v-\frac{\partial v}{\partial z}\right) n
$$

where the terms from the electron diffusion and electron recombination are neglected, since these terms are smaller than the other terms.

The results of the laboratory experiment obtained by Allibone and Meer [4] show that the streamer advances with a nearly constant velocity except just after starting from one electrode and just before arriving at another electrode. Here we assume that the velocity of the streamer within each step in lightning discharges are also nearly constant except just after starting and just before stopping by the analogy of the above experiment. If the streamer advances with a constant velocity, it is reasonable to assume that the electron density, $n$, and the electric field in front of the tip of the streamer are independent of the time on the $(\zeta, \eta$, $\xi)$ coordinate, namely $E=E(\xi)$ and $n=n(\xi)$. Letting the constant velocity of the streamer by $V$,

$$
\xi=z-V t,
$$

therefore $n=n(\xi)=n(z-V t)$ and $E=E(\xi)=E(z-V t)$. While $\alpha$ and $v$ are functions of $E$, so they are functions of $z-V t$. Thus all physical quantities in the equation (3) are functions of $z-V t$, so the differential operators in (3) can be transformed as follows :

$$
\left\{\begin{array}{l}
\frac{\partial}{\partial t}=-V \frac{d}{d \bar{\xi}^{-}} \\
\frac{\partial}{\partial z}=\frac{d}{d \xi} .
\end{array}\right.
$$

Substituting (5) into (3),

$$
-V \frac{d n}{d \xi}=-v \frac{d n}{d \xi}+\left(\alpha v-\frac{d v}{d \xi}\right) n
$$

Integrating, (6) we get

$$
\log n=\int_{C}^{\xi} \frac{\alpha v-\frac{d v}{d \xi}}{v-V} d \xi
$$

where $C$ is an integration constant.

In order that the streamer may begin to advance, a considerably strong electric field at the tip of the streamer (i.e. $\geqq 3 \times 10^{4} \mathrm{volt} / \mathrm{cm}$ at $760 \mathrm{~mm} \mathrm{Hg}$ ) must be produced by some causes [2] and has to be held at this value during the progress. It is assumed as before that the region within which the electron can ionize the 
air molecules by collisions reaches to $d \mathrm{~cm}$ from the tip of the streamer $(\xi=0)$. Denoting the electron density at the tip of the streamer, $A$, and that at $B$ by $n_{s}$ and $n_{B}$ respectively,

$$
\left\{\begin{array}{lll}
n=n_{s} & \text { at } & \xi=0 \\
n=n_{B} & \text { at } & \xi=d .
\end{array}\right.
$$

So, from (7) and (8)

$$
\int_{0}^{d} \frac{d v-\frac{d v}{d \xi}}{V-v} d \xi=\log \frac{n_{s}}{n_{B}} .
$$

As $n_{B}$ approaches zero, the right-hand side in (9) tends to infinity. While the range of integration is finite and also $\alpha v-\frac{d v}{d \xi}$ is finite, and so $V$ must tend to $v$ which is a definite value of the drift velocity of the electron in $A B$. This shows that the velocity of the pilot streamer through the virgin air is $v(\bar{v}$ may correspond to this value of $v$ whose magnitude depends on the tip field). From the above discussions, it is shown that the expression (9), by which $V$ is given, comprises the velocity of the pilot streamer as a limiting case.

In order to obtain $V$ from (9), it is necessary to know the dependence of $\alpha$ and $v$ on $E$.

In the range of $\alpha / p$ in which the lightning discharge occurs ( $p$ is the gas pressure), i.e. in the range from 40 to 60 of $\alpha / p$ (where the electric field is measured in volt $/ \mathrm{cm}$ and $p$ in atmosphere unit), the following empirical formula holds:

$$
\alpha=a E^{2}+b E+c
$$

where $a, b$ and $c$ are numerical parameters dependent on the gas pressure. In the same region of $\alpha / p, v$ is written as

$$
v=k E^{1 / 2}
$$

In cases where all collisions between the electrons and the gas molecules are completely inelastic, the theoretical value of $k$ is $\sqrt{(\pi \lambda e / 2 \mathrm{~m})}=3.6 \times 10^{6}$ in C.G.S. unit [5], which is slightly larger than the actual value, where $e$ is the electronic charge, $m$ is the electronic mass and $\lambda$ is the collision mean free path of the electron. In the case of elastic collision, the expression of $k$ has a more complex form, which gives a smaller value than the actual one. The observational results in the lightning discharge show that the reasonable value of $k$ is $10^{6}$ in C.G.S. unit [1]. So we shall use this value in this paper.

Substituting (10) and (11) into (9), we get

$$
\int_{0}^{d} \frac{a^{\prime} E^{\bar{q} / 2}+b^{\prime} E^{3 / 2}+c^{\prime} E^{1 / 2}-k \frac{d E^{1 / 2}}{d \xi}}{V-k E^{1 / 2}} d \xi=\log \frac{n_{s}}{n_{B}}
$$

where $a^{\prime}=a k, b^{\prime}=b k$ and $c^{\prime}=c k$.

If the $E(\xi)$ and $n_{s} / n_{B}$ are known, $V$ can be calculated by (12), i.e. $V$ is to be in principle written as follows: 


$$
V=\phi\left(E_{0}, E_{d}, \log \frac{n_{s}}{n_{B}}\right),
$$

where $\phi$ is some functional form, arguments $E_{0}$ are $E_{d}$ are the electric field at $\xi=0$ and $\xi=d$ respectively. In the following section, we shall calculate $V$ on the basis of the concrete form of $E(\xi)$.

\section{The volocity of the stepped streamer}

We shall calculate the velocity of the stepped streamer as an example in this section. In order to calculate the equation (12), it is necessary to know the electric field in $A B$.

In this case, the tip field may have a somewhat complex form due to the space charge before and behind the tip, but assuming that this field has the form of $Q / R^{2}$ may not lead to any serious error. So, for simplicity, we assume here that the tip field is given by the following expression:

$$
E=\frac{Q}{(r+\xi)^{2}}
$$

where $r$ is the radius of the channel and $Q$ is a constant. The electric field at the tip of the streamer in progress must be greater than $3 \times 10^{4}$ volt $/ \mathrm{cm}$ at $760 \mathrm{~mm} \mathrm{Hg}$., but may not appreciably differ from this, since the ionization and the excitation probabilities of the air molecule rapidly increase above this critical field. Therefore, the tip field may be assumed to range from $3 \times 10^{4}$ volt $/ \mathrm{cm}$ to $10^{5} \mathrm{volt} / \mathrm{cm}$. Furthermore we assume that $r=2.5 \mathrm{~m}$ following Schoncand [2], which gives values of the corresponding effective charge, $Q$, of $6.3 \times 10^{6}$ e.s.u. to $2.1 \times 10^{7}$ e.s.u.

Transforming the integral variable $\xi$ into $E$ by (13), the equation (12) becomes

$$
\frac{k \sqrt{Q}}{2} \int_{E_{d}}^{E_{0}} \frac{a E+b+c E^{-1}+\frac{E^{-1 / 2}}{\sqrt{ } Q}}{V-k E^{1 / 2}} d E=\log \frac{n_{s}}{n_{B}} .
$$

Integrating (14), we get

$$
\begin{aligned}
& {\left[\sqrt { Q } \left\{\frac{a}{k^{3}}\left(V^{3} \log u-3 V^{2} u+\frac{3}{2} V u^{2}-\frac{u^{3}}{3}\right)+\frac{b}{k}(V \log u-u)\right.\right.} \\
& \left.\left.\quad+\frac{c k}{V} \log \frac{u}{V-u}+\frac{1}{\sqrt{Q}} \log u\right\}\right]_{u_{0}}^{u_{d}}=\log \frac{n_{s}}{n_{B}},
\end{aligned}
$$

where $u=V-k E^{1,}, u_{d}$ and $u_{0}$ are the values of $u$ at $\xi=d$ and $\xi=0$ respectively. Since it is tedious to solve (15) analytically with respect to $V$, we get the relation between $V$ and $\log \left(n_{s} / n_{B}\right)$ by the numerical calculation and show it in Fig. 2, where constants $a, b$ and $c$ used are the following values (at $760 \mathrm{~mm} \mathrm{Hg}$ ) which are calculated from the experimental values [6]:

$$
a=1.40 \times 10^{-2}, \quad b=-1.99, \quad c=7.14 \times 10 .
$$

Parameters on curves represent $d$ 's, corresponding to the various values of the charge $Q$. Using $10^{14} / \mathrm{cm}^{3}$ and $10^{10} / \mathrm{cm}^{3}$ which are the accepted values of $n_{s}$ and $n_{B}$ 


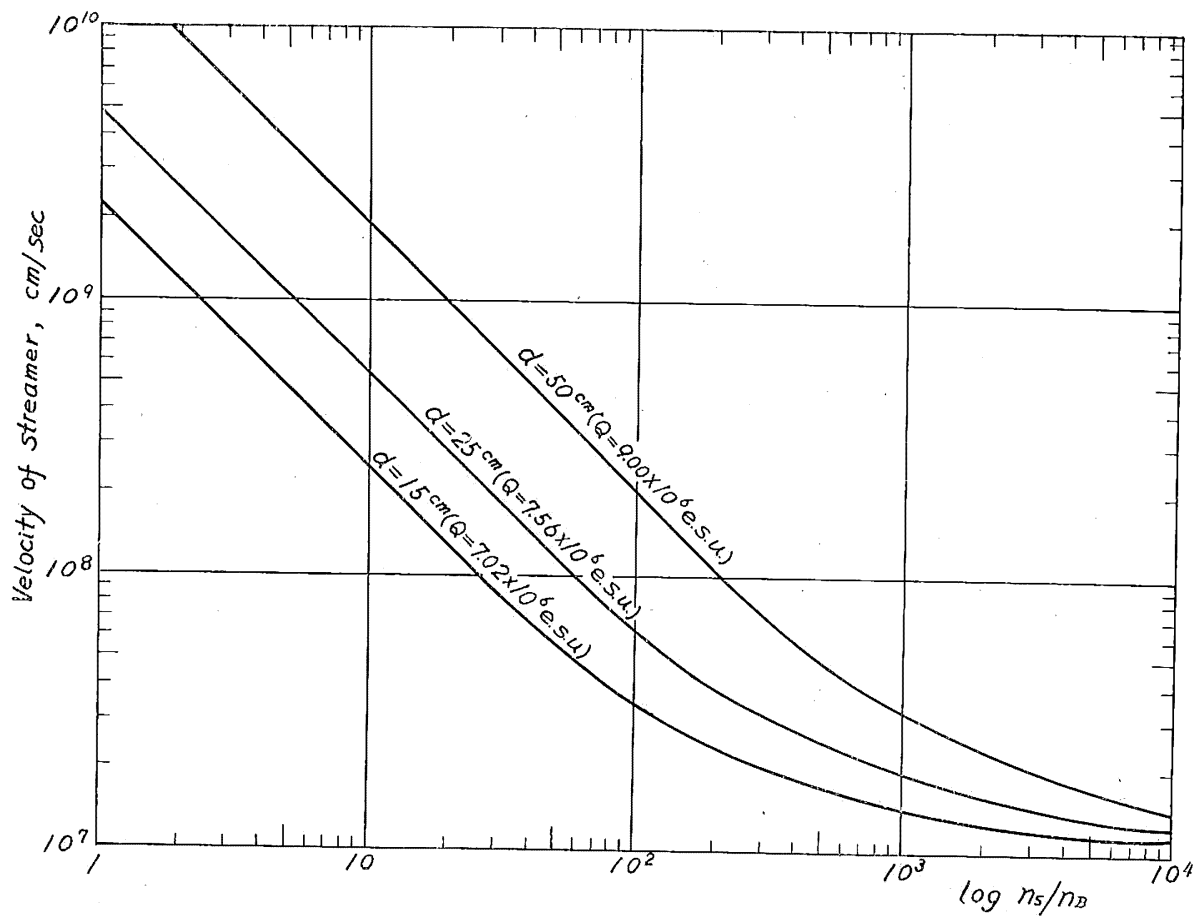

Fig. 2. The relation between $V$ and $\log \left(n_{s} / n_{B}\right)$ for various values of the effective charge $Q$.

respectively, we get the velocity of the streamer as $2.8 \times 10^{8} \mathrm{~cm} / \mathrm{sec}, 5.9 \times 10^{8} \mathrm{~cm} / \mathrm{sec}$ and $2.2 \times 10^{9} \mathrm{~cm} / \mathrm{sec}$ according to the magnitudes of the $Q$ given above. The last value agrees fairly well with the velocities hitherto observed.

\section{Discussion}

We have obtained the expression on the velocity of the streamer, in which the time variation of the electron density in the channel is taken account of, in place of the incomplete expression (1) obtained so far, but it is a slight improvement of (1). In section 3 , the electric field required for advancing the stepped streamer is assumed as $Q /(r+\xi)^{2}$, which is to be improved on. To do this, we shall consider the elementary processes of the collisions between the electrons and the air molecules in more detail in future. The formation of the field, the electric current carried through the channel and the constancy of the velocity of the streamer have to be derived on the basis of the elementary processes, though this is difficult.

Acknowledgement - The authors wish to express their hearty thanks to Professor K. Honda and the members of the Geoelectric and Geomagnetic Laboratory, Meteorological Research Institute, for their encouragement and helpful advice throughout the present work. 


\section{References}

[1] Schonland, B.F.J., 1938: Progressive Lightning (IV)-The Discharge Mechanism. Proc. Roy. Soc. A. 164, p. 132.

[2] Schonland, B.F.J., 1953: The Pilot Streamer in Lightning and the Long Spark. Proc. Roy. Soc. A. 220, p. 25.

[3] Loeb, B.L., 1939: Fundamental Processes of Electric Discharge in Gases, p. 540.

[4] Alujbone, T.E. and Merk, J.M., 1938: The Development of the Spark Discharge. Proc. Roy. Soc. A. 166, p. 97.

[5] Compron, K.T., 1923: On the Motions of Electrons in Gases. Phys. Rev. 22, p. 333.

[6] Meek, J.M. and Cragas, J.D., 1953: Electrical Breakdown of Gases. Oxford, p. 58. 